\title{
SRC "Planeta" United System for Distributed Data Handling: Actual Features and Future Evolution
}

\author{
Mikhail A. Burtsev ${ }^{1}$, Oleg E. Milekhin ${ }^{2}$, Lyubov S. Kramareva ${ }^{3}$, Valeriy N. Antonov ${ }^{4}$, Ivan V. Balashov ${ }^{1}$, \\ Alexandr V. Kashnitskiy ${ }^{1}$, Evgeny A. Loupian ${ }^{1}$, Alexey M. Matveev ${ }^{1}$, \\ Andrey A. Proshin ${ }^{1}$, Sergey A. Uspenskiy ${ }^{2}$ \\ ${ }^{11}$ Space Research Institute, Russian Academy of Sciences, Moscow, Russia \\ burcev@d902.iki.rssi.ru \\ ${ }^{2}$ SRC "Planeta", Moscow, Russia \\ milekhin@planet.iitp.ru \\ ${ }^{3}$ Far-Eastern Center of SRC "Planeta", Khabarovsk, Russia \\ kramareva@dvrcpod.ru \\ ${ }^{4}$ Siberian Center of SRC "Planeta", Novosibirsk, Russia \\ avn@rcpod.siberia.net
}

\begin{abstract}
This paper describes the results of five years operation of the SRC "Planeta" united system for distributed data handling, its actual features and future evolution trends. During this time period the system has grown into a complex data handling instrument providing the solution of many various tasks including hydrometeorological monitoring, emergency monitoring etc. with the system's resources and data processing and analysis tools only. All these resources are available on the web. Now the system is a distributed modular web-GIS application integrated with very large Earth Remote Sensing online data archives. Furthermore, the system provides online data services for the "IKI-Monitoring" shared computing center and large amount of applied information system thus being one of the main near real-time satellite data providers in Russia.
\end{abstract}

Keywords: satellite data, distributed systems, very large data archives, space hydrometeorology, Earth Remote Sensing systems 


\title{
ОБЪЕДИНЕННАЯ СИСТЕМА РАСПРЕДЕЛЕННОЙ РАБОТЫ С ДАННЫМИ ЦЕНТРОВ ПРИЕМА НИЦ "ПЛАНЕТА": ТЕКУЩИЕ ВОЗМОЖНОСТИ И ПЕРСПЕКТИВЫ РАЗВИТИЯ
}

\author{
Буриеев М.А. ${ }^{1}$, Милехин О.Е. ${ }^{2}$, Крамарева Л.С. ${ }^{3}$, Антонов В.Н. ${ }^{4}$, Балашов И.В. ${ }^{1}$,

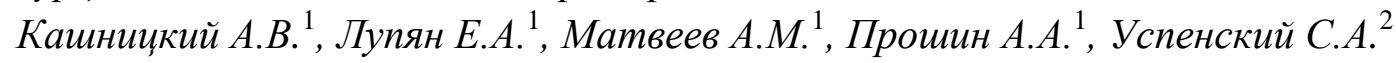 \\ ${ }^{1}$ Институт космических исследований РАН, Москва, Россия \\ burcev@d902.iki.rssi.ru \\ ${ }^{2}$ НИЦ «Планета», Москва, Россия \\ milekhin@planet.iitp.ru \\ ${ }^{3}$ Дальневосточный центр НИЦ «Планета», Хабаровск, Россия \\ kramareva@dvrcpod.ru \\ ${ }^{4}$ Сибирский центр НИЦ «Планета», Новосибирск, Россия \\ avn@rcpod.siberia.net
}

\begin{abstract}
Настоящая статья посвящёна результатам работы Объединённой системы работы с данными региональных центров ФГБУ «НИЦ «Планета» за пять лет, прошедшие с момента её ввода в эксплуатацию, описанию её текущих возможностей, а также анализу основных направлений её развития. За это время система развилась в сложный, комплексный инструмент работы с данными, позволяющий решать задачи гидрометеорологического мониторинга, мониторинга чрезвычайных ситуаций и многие другие задачи только за счёт ресурсов и инструментов обработки и анализа данных самой системы, доступных через веб. На сегодняшний день система представляет собой распределённую, модульную вебГИС, совмещённую со сверхбольшими архивами данных ДЗЗ прямого доступа. Кроме того, система предоставляет онлайн-сервисы данных для ЦКП "ИКИ-Мониторинг" и большого количества прикладных информационных систем, являясь одним из основных источников оперативной спутниковой информации в России.
\end{abstract}

Ключевые слова: спутниковые данные, распределённые системы, сверхбольшие архивы данных, космическая гидрометеорология, системы ДЗ3

\section{Введение}

ФГБУ «НИЦ «Планета» - ведущая организация Росгидромета по обеспечению космического мониторинга в целях решения задач оперативного гидрометеорологического обеспечения, мониторинга глобальных изменений Земли и её климата, мониторинга загрязнений окружающей среды, мониторинга чрезвычайных ситуаций и многих других. В состав НИЦ «Планета» входит сеть из трёх региональных центров (Европейский, гг. Москва, Обнинск, Долгопрудный, Сибирский, г. Новосибирск и Дальневосточный, г. Хабаровск), а также более 70 стационарных и 
мобильных автономных пунктов приёма информации в России, Антарктиде и на морских судах. Эта сеть обеспечивает полное регулярное покрытие спутниковой информацией всей территории России и Европы. Для обеспечения возможности прозрачной, единообразной работы с данными всех трёх региональных центров была совместно разработана специалистами НИЦ «Планета» и ИКИ РАН и введена в эксплуатацию в 2013 году [1] Объединённая система работы с данными региональных центров ФГБУ «НИЦ «Планета». Она предназначена для обеспечения специалистов возможностью оперативного прозрачного доступа к распределённым архивам данных, а также их последующей обработки и совместного анализа. На текущий момент система обеспечивает работу с данными более чем 15 отечественных и зарубежных КА, в том числе КА серий «Метеор-М», «Канопус-В» и «Ресурс-П».

\section{Предпосылки к созданию системы и её задачи}

Ежесуточно в центрах НИЦ «Планета» принимается и обрабатывается более 1,3 ТБ спутниковой информации с 16 зарубежных и отечественных КА ДЗ3. На основе этой информации выпускается около 500 видов информационной продукции для более чем 500 потребителей федерального и регионального уровней. Кроме того, объемы получаемой и предоставляемой информации ежегодно увеличиваются, что соответствует общемировым трендам [2], [3]. Исторически сложилось так, что в каждом из трёх региональных центров существовали свои системы обработки, ведения архивов и распространения данных, а также свои стандарты формирования выходной информационной продукции. Это привело к тому, что для работы с данными каждого центра приходилось создавать собственные механизмы и к серьёзному усложнению совместного анализа и обработки этих данных. Все это в сочетании с быстрым развитием веб-технологий привело к необходимости и возможности создания единого, универсального средства работы с данными центров приёма, обработки и распространения, охватывающее все этапы их жизненного цикла от получения со станции до предоставления их специалисту.

Основные задачи создаваемой системы включали в себя:

- Предоставление возможности однотипной, прозрачной работы с данными всех центров НИЦ «Планета»;

- Унификация и автоматизация систем обработки данных в различных центрах НИЦ «Планета»;

- Обеспечение возможности распределенной работы с архивами спутниковых данных различных центров;

- Предоставление инструментов для анализа спутниковых данных и информационных продуктов, получаемых на их основе в различных центрах;

- Поддержка унифицированной схемы онлайн-взаимодействия с различными системами дистанционного мониторинга.

Для решения этих задач была разработана архитектура системы, представленная на рис. 1.

В рамках этой архитектуры были созданы единая территориально распределенная подсистема архивации данных, обеспечивающая возможность работы как с информацией уровня L1B, так и с различными информационными продуктами более высоких уровней, унифицированные комплексы обработки и архивации спутниковых данных в центрах НИЦ «Планета», система удаленного доступа к распределенных архивам спутниковых данных, а также система контроля и управления, охватывающая все компоненты системы. Кроме того, в рамках системы была разработана максимально унифицированная технология онлайн-взаимодействия системы с различными внешними информационными системами и центрами. 


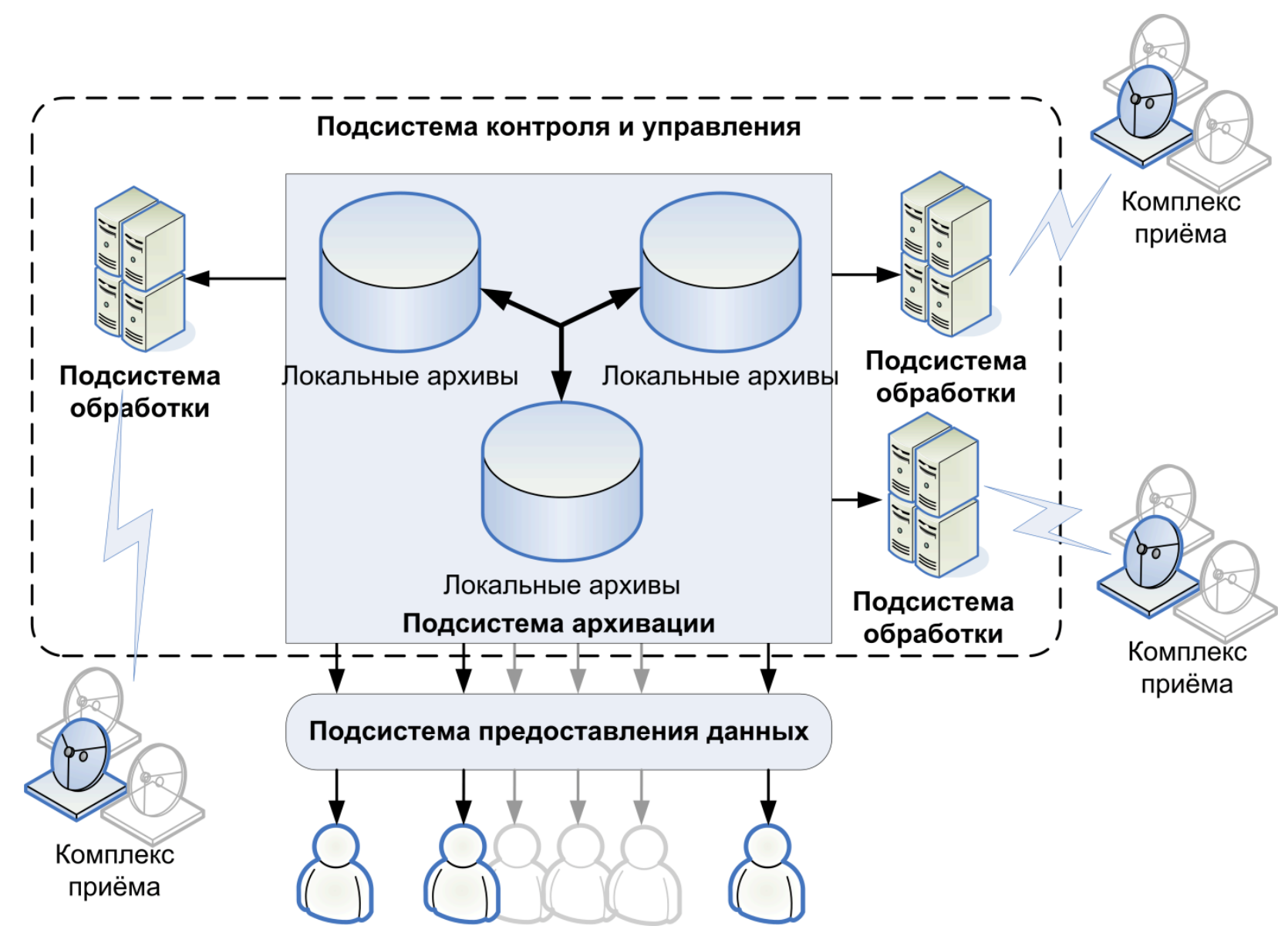

Рис. 1. Архитектура системы

\section{Состав и возможности системы}

Основными компонентами системы являются подсистемы обработки, архивации и доступа к данным, расположенные в каждом центре. Подсистема обработки данных включает в себя набор серверов диспетчеризации данных, обеспечивающих обмен данными между комплексами приёма и подсистемой архивации, а также набор универсальных станций обработки данных.

Подсистема архивации включает в себя набор локальных архивов данных, хранящих исходные данные и результаты обработки, полученные в каждом центре. Эти локальные архивы объединяются в единый логический архив на уровне метаданных - при изменении наполнения одного из локальных архивов метаданные синхронизируются во все узлы (центры) системы, что позволяет иметь в каждом центре полную информацию о содержимом всего единого архива. Это позволяет повысить быстродействие системы, а также обеспечить дополнительную отказоустойчивость. Архивы реализованы на базе технологии UNISAT [4], которая обеспечивает единообразное ведение и построение распределённых архивов данных Д3З и даёт возможность работы с т.н. «виртуальными» продуктами - данными, полученными в результате обработки «на лету» и результатами интерактивной обработки.

Подсистема доступа к данным включает в себя пользовательские веб-интерфейсы для работы с данными и программные интерфейсы (API) для обеспечения обмена данными с внешними системами. Пользовательские картографические интерфейсы построены на базе технологии GEOSMIS [5], обеспечивающей создание сложных картографических вебинтерфейсов работы с данными ДЗ3. Она позволяет реализовать как операции доступа к данным, так и операции анализа и интерактивной обработки данных. Кроме того, она обеспечивает модульность и гибкость создаваемых интерфейсов. Картографические интерфейсы 
предоставляют как стандартные для онлайн-систем работы с данными Д3З функции поиска данных по разным критериям, в том числе времени, пространству, типу и источнику, их просмотра и сохранения для дальнейшей работы, так и инструментарий интерактивной обработки и анализа данных [6]. Реализованные инструменты включают в себя инструменты сравнения данных (шторка и прозрачность), цветосинтез, цветокоррекцию, коррекцию гистограмм, в т.ч. разновременных данных, классификацию данных, в т.ч. с обучением и без, алгебру изображений, инструмент построения одно- и двумерных гистограмм по отобранным данным, расчёт высоты облачности, структурный анализ, инструмент коррекции привязки.

Также система обеспечивает онлайн-обмен данными с различными специализированными системами и центрами дистанционного мониторинга. В число этих систем входят:

- ЦКП «ИКИ-Мониторинг» [7] / информационные системы РАН семейства «ВегаСозвездие»;

- Информационная система дистанционного мониторинга Рослесхоза (ИСДМ-Рослесхоз);

- Система дистанционного мониторинга вулканической активности Камчатки и Курил (VolSatView);

- Российский сегмент системы глобального агрометеорологического мониторинга (GEOGLAM);

- Геопортал Роскосмоса;

\section{Заключение}

В ходе создания и постоянного развития системы удалось реализовать однотипные схемы обработки, хранения и представления спутниковой информации во всех центрах НИЦ «Планета», беспечить возможность «прозрачной» работы с распределенными архивами и вычислительными ресурсами различных центров и организовать возможность онлайнвзаимодействия с различными информационными системами дистанционного мониторинга. Это привело к тому, что был создан новый инструмент распределенной работы со спутниковой информацией для решения задач гидрометеорологии и мониторинга окружающей среды, причём инструмент как для работы экспертов в интерактивном режиме - модульная Web-GIS, совмещённая со сверхбольшими архивами данных ДЗ3 прямого доступа, так и для работы специализированных систем, направленных на решение конкретных задач - один из главных провайдеров онлайн-сервисов оперативных спутниковых данных в России. Естественно, развитие системы постоянно продолжается по мере роста спутниковых группировок ДЗ3 и совершенствования технологий работы с данными, и основными направлениями развития являются регулярное расширение доступной пользователям информации по мере роста орбитальных группировок КА ДЗЗ и увеличение состава и возможностей инструментария работы с данными для создания полноценного рабочего места метеоролога на базе Web-GIS.

Работы по развитию элементов системы в настоящее время ведутся в рамках проектов Росгидромета, Роскосмоса, РАН (тема "Мониторинг") и РФФИ (проекты 13-07-00513, 13-0712017-офи-м, 13-07-12116-офи-м-2011). 


\section{References}

[1] Loupian E.A., Milexin O.E., Antonov V.N., Kramareva L.S., Bourtsev M.A., Balashov I.V., Tolpin V.A., Solovyev V.I. System of operation of joint information resources based on satellite data in the Planeta Research Centers for Space Hydrometeorology, Russian Meteorology and Hydrology, 2014, Vol. 39, Issue 12, pp. 847-853. DOI: $10.3103 /$ S1068373914120103.

[2] Mirko Albani, Long Term Data Preservation: status of activities and future ESA programme, Presented at GSCB Workshop 2012 ESA/ESRIN, Frascati 6-7 June 2012. URL:

https://earth.esa.int/documents/1656065/1664726/8-LTDP_activities_future.pdf.

[3] Loupian E.A., Bourtsev M.A., Proshin A.A., Kobets D.A. Evolution of remote monitoring information systems development concepts, Sovremennye problemy distantsionnogo zondirovaniya Zemli iz kosmosa, 2018, Vol. 15, No. 3, pp. 53-66. DOI: 10.21046/2070-7401-2018-15-3-53-66.

[4] Proshin A.A., Loupian E.A., Balashov I.V., Kashnitskiy A.V., Bourtsev M.A. Unified satellite data archive management platform for remote monitoring systems development, Sovremennye problemy distantsionnogo zondirovaniya Zemli iz kosmosa, 2016, Vol. 13, No. 3, pp. 9-27.

[5] Tolpin V.A., Balashov I.V., Efremov V. Yu., Loupian E.A., Proshin A.A., Uvarov I.A., Flitman E.V. The GEOSMIS System: Developing Interfaces to Operate Data in Modern Remote Monitoring Systems, Sovremennye problemy distantsionnogo zondirovaniya Zemli iz kosmosa, 2011, V. 8, No. 3, pp. 93-108.

[6] Kashnitskiy A.V., Loupian E.A., Balashov I.V., Konstantinjva A.M. Technology for Designing Tools for the Process and Analysis of Data from Very Large Scale Distributed Satellite Archives, Atmospheric and Oceanic Optics, 2016, Vol. 30, Issue 1, pp. 84-88. DOI: 10.1134/S1024856017010080.

[7] Loupian E.A., Proshin A.A., Bourtsev M.A., Balashov I.V., Bartalev S.A., Efremov V. Yu., Kashnitskiy A.V., Mazurov A.A., Matveev A.M., Sudneva O.A., Sychugov I.G., Tolpin V.A., Uvarov I.A. IKI center for collective use of satellite data archiving,processing and analysis systems aimed at solving the problems of environmental study and monitoring, Sovremennye problemy distantsionnogo zondirovaniya Zemli iz kosmosa, 2015, Vol.12, No 5, pp. 263-284. 